\title{
Modeling and Resolution of the Allocation Problem of the Time Slots in Dakar Airport
}

\author{
Youssouf Ahamada ${ }^{1}$, Salimata G. Diagne ${ }^{1}$, Amadou Coulibaly ${ }^{1}$, Déthié Dione ${ }^{1}$, N'dogotar Nlio $^{2} \&$ Youssou Gningue \\ ${ }^{1}$ Cheikh Anta Diop University, Dakar, Senegal, Tchad \\ ${ }^{2}$ University of Sarh, University of Laurentia \\ ${ }^{3}$ Sudbury, Canada \\ Correspondence: Déthié DIONE, Cheikh Anta Diop University, Dakar, Sngal. E-mail: dethidione79@ gmail.com
}

Received: February 9, 2017 Accepted: April 12, 2017 Online Published: May 18, 2017

doi:10.5539/jmr.v9n3p30 URL: https://doi.org/10.5539/jmr.v9n3p30

\begin{abstract}
In this article, we proposed a programming linear model in integer numbers(PLIN) for the optimal allocation of the time slots in the international Leopold Sedar Senghor airport of Dakar (L.S.S). The slots are specific allocated periods which allow an aircraft to land or take off in a saturated airport. Their attribution depends on the configuration of the airport, more particularly on its capacity. We maximize the confirmed demand in each slot and take the number of aircrafts and the number of manageable passengers with an optimal quality service into account. We used the CPLEX software so that to test the effectiveness of the linear model. Firstly, in the proposed model linear in integer numbers, any unmet demand was isolated. Secondly, the rejected demands by introducing a model and an algorithm of resolution based on the dynamic programming.
\end{abstract}

Keywords: optimization, dynamic programming, allocation, times slot.

\section{Introduction}

The airport managers devote the airports (2007) at the service of the airline companies. These airports are composed of subsystems with which are associated a level of quality and service. The international Leopold Sedar Senghor airport of Dakar (LSS) is facing problems of congestion, i.e. the demand is greater than the supply. Indeed, this phenomenon is due to the insufficiency of its capacity. This is justified both by the insufficient capacity caused by the sharp increase in air traffic over the past few years and by the transport demand which is subject to large fluctuation over time. Therefore, the Blaise Diagne Airport is under construction to cope with the problem caused by capacity but its completion takes time in view of the financial, environmental difficult, etc, raised. Thus, until the problems are resolved, it is question of exploiting in an optimal way the L.S.S airport of Dakar. The time slots depend on the capacity of the airport.

The aim of this article is to help the coordinator of the L.S.S airport of Dakar to allocate these time slots by using a mathematical model based on the linear programming of integer numbers which will be then solved. Several studies were made on the problem of allocation of the airport slots, but in all these studies, the capacity of the airport is related with that of the runway. Thus in 1975, at STBA ${ }^{1}$ a first model resulting from the queuing theory was proposed (1975). In 1993 (Eugene P Gilbo., 1993) and in 2003 (Eugene Gilbo, 2003) Gilbo used a dynamic program and an estimation method is determine the runway capacity. In 2007, Alidou SINARE (2007) determined the capacity of the air of the airport traffic of Dakar by a method of approximation and evaluation. ABDELLAH Idrissi (Idrissi, Abdellah, \& Li, Chu., 2005) modeled the problem of allocation of the capacity of the posts of parkings in the form of a CSPA ${ }^{2}$. All these authors contributed to solving of the problems of allocation in the airport slots by considering the capacity of the runway as the single possible constraint.

Therefore our contribution in this article takes into account the runway and the terminal of the passengers. It is a question of maximizing the number of demands to affect in a slot under the constraint of capacity. The Software Cplex was used to test the model. Indeed, with the proposed model, any unmet demand was isolated. We integrated thus, rejected demands by introducing a model and an algorithm of resolution based on the dynamic programming.

This paper is organized as follows: We have started with the introduction of the section (1), in the section (2) we described the problem of allocation in the slots and we have specified the main constraints of these slots. The proposed model and the introduced dynamic model of programming are described in the section (3). In the section (4), We will speak about the methods of resolutions. And finally, the conclusion in the section (5).

\footnotetext{
${ }^{1}$ Air Base for Technical Services, July 1975.

${ }^{2}$ Airport Problems of Satisfaction Constraint
} 


\section{Description of the Allocation problem for the Airport's slots}

The allocation problem of the Slots can be analyzed in various domains, and we shall study these problems more specifically: indeed, there is not any generic model for such problem. Every domain has its own constraints, and the degree of the problem itself is linked to the number of constraints. The airport capacity is a heavy constraint for the attribution of the time slots

\subsection{Airport Capacity}

The capacity is a limit which, when exceeded, will affect the fluidity of the airport operations and will not correspond to the level of quality of the preset service (2007) fixed by the ICAO(International aviation of airline company ) standards. It is the number of demands which can be satisfied at the peak hour by the whole of the airport installations. This capacity shall respect the safety requirements and that of the environment. In the context of this article, the capacity of the airport is composed of:

- Capacity of the air movements:

The critical operations are located on the runway, the aforementioned becomes the bottleneck of the air movement. The capacity of the runway is often mistaken with that of the capacity of the air movements. Indeed (Stoica, 2004), the capacity of the runway is defined as the number of movements which can occur on the runway for a given time slot.

- The capacity of the passengers terminal, it is the number of passengers whom the terminal can run out with the quality of service required by the ICAO, also the treatment of the passengers in a given slot is taken into account (Service technique de laviation civil). It is composed of the capacity on arrival and departure.

\subsection{Allocation Problem of the Slots:}

Because of the congestion or saturation of an airport, the time slots constitute an invaluable and coveted resource by the airline companies. A time slot is an authorization to use the infrastructures of a coordinated airport ${ }^{3}$ on a precise date and hour, according to the attribution made by the coordinator (Nathalie Lenoir, 2004). Each country has its coordinator and the market of the slots takes place twice per year, in summer and winter. The airport coordinator allots the slots on the basis of these two seasons and the rules of the international aviation of airline company (ICAO) into force.

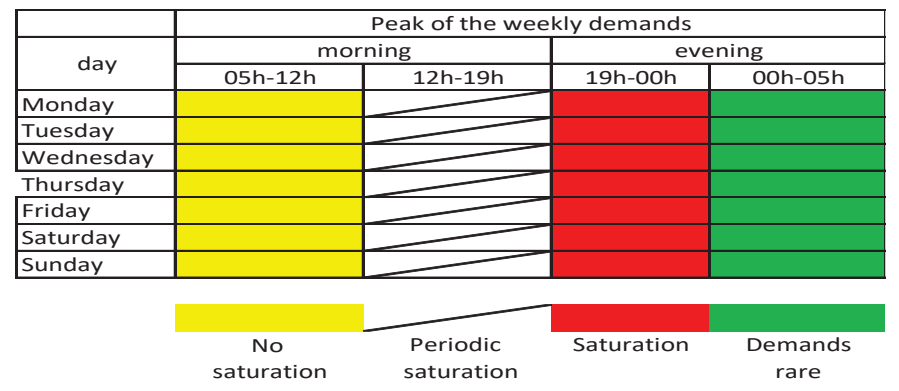

Figure 1. Peak of the weekly demands

The demand for air transport in the Dakar airport is prone to many fluctuations: during the day (peaks the morning as well as the evening), in a year, significant demands in winter than summer, see Fig-1.

However, the airline companies provide the airport coordinator the relevant information which constitute the demand: whether the peak of the morning or evening, the season (winter or summer), the preferential hour of landing or takeoff in the peak, type of plane used in the season, the number of seats that the plane contain, etc. Before confirming the demands of the companies, the coordinator asks himself a certain number questions, on average, how many demands to confirm in a slot? For one day? For one week or season? etc. To answer these questions the coordinator must take the constraint of capacity during the concerned periods into account. When a demand cannot be satisfied in a slot, the coordinator communicates the reasons to the applicant and he indicates another one which may be the nearest slot to him.

Therefore, the coordinator establishes the planning of the airport (diagram of slot) for the different confirmed demands of the airline companies, and this planing will be refined in real time by the controllers.

\footnotetext{
${ }^{3}$ An airport where, to land or take off, an airline company or all other aircraft must have been allotted a time slot by a coordinator, allowing for exception: the flights of State, the emergency landings and humanitarian flights.
} 


\section{Modeling of the Problem}

We raise the following hypothesis:

$\diamond H_{1}$ : The number of the plane seats declared by the company corresponding to the number of passengers of the plane, this is to say that all the planes are supposed to be full.

$\diamond \mathrm{H}_{2}$ : The horizon of optimization is cut out in windows of time, and each window indicates a time slot as the figure-2 shows it below.

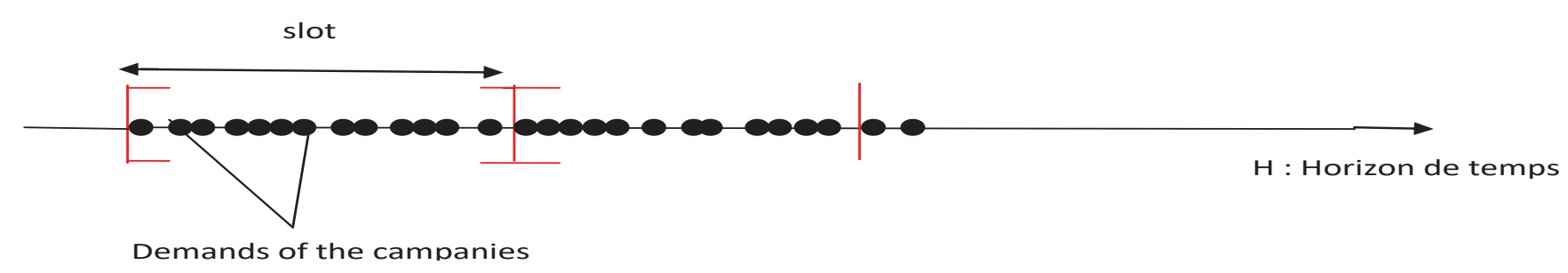

Figure 2. Optimization horizon

$\diamond H_{3}$ : The passengers terminal contains only two reception rooms, one on arrival and another for the departure. In oder to be a quality of service, we fixed the number of manageable passengers, on arrival and departure for a slot in this terminal. Beyond this capacity the service is not optimal.

\subsection{Mathematical terminology:}

I: All the slots of the optimization horizon, indexed by $\mathrm{i}=1,2, \ldots \mathrm{i}, \ldots \mathrm{N}$

A: Maximum capacity by number of planes of the runway for a slot on arrival.

D: Maximum capacity by number of plane of the runway for a slot on departure.

$C_{a}$ : Maximum number of manageable passengers on arrival in a slot.

$C_{d}$ : Maximum numbers of manageable passengers on departure in a slot.

$A_{i}$ : All the planes which ask to come to the slot $i$.

$D_{i}$ : All the planes which ask to leave the slot $i$.

$p_{i k}$ : The number of passengers of the plane $k$, on arrival, for the slot $i$.

$q_{i l}$ : The number of passengers of the plane $l$, on departure, of the slot i.

\section{Variables}

We indicate by $x_{i k}$ and $y_{i l}$ the variables of decisions.

$$
x_{i k}= \begin{cases}1 & \text { if the demand of the plane } k \text { at arrived for a slot } i \text { is confirmed } \\ 0 & \text { otherwise }\end{cases}
$$

$P_{i}$ : total number of the confirmed passengers at the arrival slot $i$.

$$
\begin{gathered}
\qquad P_{i}=\sum_{k \in A_{i}} p_{i k} x_{i k} \quad \forall i \in I \\
y_{i l}= \begin{cases}1 & \text { if the demand of the plane } l \text { at departure for the slot } \mathrm{i} \text { is confirmed. } \\
0 & \text { otherwise }\end{cases}
\end{gathered}
$$

$Q_{i}$ : total number of the confirmed passengers at the slot $i$ of departure.

$$
Q_{i}=\sum_{l \in D_{i}} q_{i k} y_{i l} \quad \forall i \in I
$$




\subsection{Mathematical Formulation}

To work out the slot diagram of the international Lopold Sedar Senghor airport in one season, we propose to establish it in a way of satisfying the maximum demands, and this brings us to formulate the following problem $\mathcal{P}_{1}$.

$$
\max _{i \in I} \sum_{k \in A_{i}} x_{i k}+\sum_{l \in D_{i}} y_{i l}
$$

Under the constraints:

$$
\begin{gathered}
\sum_{k \in A_{i}} x_{i k} \leq A \quad \forall i \in I \\
\sum_{l \in D_{i}} y_{i l} \leq D \quad \forall i \in I \\
\sum_{k \in A_{i}} p_{i k} x_{i k} \leq C_{a} \quad \forall i \in I \\
\sum_{l \in D_{i}} q_{i l} y_{i l} \leq C_{d} \quad \forall i \in I \\
x_{i k} \in\{1,0\} ; \quad y_{i l} \in\{1,0\} \quad \forall i \in I
\end{gathered}
$$

Constraints (4),(5), (6) and (7) are constraints of capacity.

Constraints (4) and (5) make sure that the accepted number of demands on arrivals and departures do not exceed respectively the maximum capacity on arrival and departure. Constraints (6) and (7) mean respectively that the sum of the passengers of all the accepted requests on arrival and departure is lower than the maximum number of manageable passengers in a slot on arrival and departure. The constraints (8) are constraints of integrity.

On the assumption that the denied demands in $i$ must be accepted in $i+1$. We introduce two logical constraints (9) and (10) stipulating that if the demand is denied in $i$ it must be accepted in slot $i+1$.

$$
\begin{array}{ll}
x_{i k}+x_{i+1 k}=1 & \forall i \in I \\
y_{i l}+y_{i+1 l}=1 & \forall i \in I
\end{array}
$$

Actualy, these denied demands in $i-1$ are added dynamically to those who formulated their requests in the slot $i$. Thus we have: $X_{i-1}+\sum_{k \in A_{i}} x_{i k}$ and $Y_{i-1}+\sum_{l \in D_{i}} y_{i l} \quad \forall i \in I$ where, respectively represents the number of accepted demand on arrival and departure in the slot $i$ with

$$
X_{i}=\left|A_{i}\right|-\sum_{k \in A_{i}} x_{i k} \quad \forall i \in I
$$

and

$$
Y_{i}=\left|D_{i}\right|-\sum_{l \in D_{i}} y_{i l} \quad \forall i \in I
$$

$X_{i}$ and $Y_{i}$ respectively represents the number of demand on arrival and departure in a non-accepted slot i. The total confirmed number of passengers on arrival and departure of a slot $i$ is:

$$
P_{i}=P_{i-1}^{r a}+\sum_{k \in A_{i}} p_{i k} x_{i k} \quad \forall i \in I
$$

and

$$
Q_{i}=Q_{i-1}^{r d}+\sum_{l \in D_{i}} q_{i l} y_{i l} \quad \forall i \in I
$$

Indeed, $P_{i-1}^{r a}$ and $Q_{i-1}^{r d}$ : stands respectively for the total number of passengers of the non-accepted demands on arrival and departure for the slot $i-1$. The problem $\mathcal{P}_{1}$ is thus reformulated in $\mathcal{P}_{2}$ and we have:

$$
\max _{i \in I}\left\{X_{i-1}+\sum_{k \in A_{i}} x_{i k}+Y_{i-1}+\sum_{k \in D_{i}} y_{i k}\right\}
$$

under the constraints:

$$
X_{i}=\left|A_{i}\right|-\sum_{k \in A_{i}} x_{i k} \quad \forall i \in I
$$




$$
\begin{gathered}
Y_{i}=\left|D_{i}\right|-\sum_{k \in D_{i}} x_{i k} \quad \forall i \in I \\
X_{i-1}+\sum_{k \in A_{i}} x_{i k} \leq A \quad \forall i \in I \\
Y_{i-1}+\sum_{k \in D_{i}} y_{i k} \leq D \quad \forall i \in I \\
P_{i-1}^{r a}+\sum_{k \in A_{i}} p_{i k} x_{i k} \leq C_{a} \quad \forall i \in I \\
Q_{i-1}^{r d}+\sum_{l \in D_{i}} q_{i k} y_{i l} \leq C_{d} \quad \forall i \in I \\
x_{i k}+x_{i+1 k}=1 \quad \forall i \in I \\
y_{i l}+y_{i+1 l}=1 \quad \forall i \in I \\
x_{i k} \in\{1,0\} ; \quad y_{i l} \in\{1,0\} \quad \forall i \in I \\
X_{i} \geq 0 \quad Y_{i} \geq 0 \quad \forall i \in I
\end{gathered}
$$

\section{Resolution}

For the resolution of the linear program in integer numbers PLIN, we used the software CPLEX and we tried the model in three different slots. In the case of dynamic programming, we worked out an algorithm of resolution.

\subsection{Simulations and Results}

For simulation, we used the Cplex software for the linear program in integer numbers, with a computer whose characteristics are as follows: Processor:Intel(R) core(TM)i5-6200U CPU @2.30GHz 2,40GHz Memory installed (RAM):8.00Go (7.90Go usable)

We used real data extracted from the time diagram of summer 2015 of the national agency of the Senegal civil aviation and meteorology (ANACIM). We took down all the arrivals and departures requests of the Tuesday between $19 \mathrm{~h}$ and $23 \mathrm{~h}$, thus, they are summarized in the following table.

In the slot of ] $19 h 20 h$, we have registered 5 arrivals requests and only 3 of them had the confirmation in the slot. In the slot of ] $20 h 21 h$, we have 4 arrivals requests and 2 demands of departure, but only one arrival demand was refused in the slot.

In the slot of [22h 23h], we have 3 arrivals requests and 4 demands of departure. Only two demands were not confirmed in the slot. We have summarized the solutions obtained by the Cplex in the following table. Interpretation

In order to be an optimal quality of service on the runway and the passengers terminal, the linear program model in integer numbers gives the results of the above table Indeed, from the confirmed demand, the coordinator will do the planning by respecting the preferential hours in the slots. By contrast, there are two possibilities for the non-confirmed demands.

1. whether the coordinator proposes the company to serve another airport with his preferential hour (in case there are two airports)

2. whether the coordinator proposes the company to serve another slot with another allotted hour.

This last case, will be the subject of the dynamic programming model. In order to take into account these unmet demands with the demanded slot, we supplemented the PLIN model by the dynamic programming, and an algorithm of resolution is established in the section (4)

\section{Conclusion}

In this article, we proposed a linear model of programming in integer number to address the issue of allocation slots. It maximizes the number of demand to be confirmed on arrival and departure by taking into account the runway capacity and the manageable number of passengers in the terminal of a given slot. We introduced logical constraints on arrival and departure, imposing that each unmet demand in a slot must be in the following slot. We used the dynamic programming and we worked out an algorithm to solve the model of the suggested dynamic programming. We tried the model by considering three slots of one hour of amplitudes with the Cplex. It turns out that certain demands should not be accepted in the needed slots so as to ensure the quality of service. We plan to simulate the dynamic programming in order to supplement the PLIN so as to work out the daily planning (or seasonal) of the Dakar airport. It will be refined in real time by the controllers. We hope in an immediate future to distinguish the set of priorities of the planes or the companies. 


\begin{tabular}{ccccc}
\hline crneau & \multicolumn{2}{c}{ Dde } & \multicolumn{3}{c}{ Passagers } \\
\hline & Arrive & Dparts & $P_{i}$ & $Q_{i}$ \\
\hline \multirow{3}{*}{ KP97 } & KE & - & 120 & - \\
]19h, 20h] $]$ & AEA132 & - & 186 & - \\
& IST/NK & - & 102 & - \\
& AF718/9 & - & 381 & - \\
& AF718/9 & - & 309 & - \\
\hline & LH826 & - & 0 & - \\
& AF718/9 & - & 381 & - \\
$i=2$ & SN206 & - & 288 & - \\
]20h, 21h] & DN64 & - & 56 & - \\
& - & KP028 & - & 120 \\
& - & NST/NK & - & 102 \\
\hline \hline crneau & Dde & & Passagers \\
\hline & Arrive & Dparts & $P_{i}$ & $Q_{i}$ \\
\hline & DN64 & - & 120 & - \\
$i=3$ & - & KP97 & - & 120 \\
]22h 23h] & - & AF718/9 & - & 381 \\
& - & AF718/9 & - & 309 \\
& - & AF718/9 & - & 381 \\
& QC376 & - & 131 & - \\
& IB69 & - & 289 & \\
\hline ]23h 24h] & - & SS990/1 & - & 304 \\
& AT501 & - & 236 & - \\
& WC & - & 124 & \\
& - & QC376 & - & 131 \\
\hline & & & &
\end{tabular}

\begin{tabular}{cccc}
\hline i & Ddes & \multicolumn{2}{c}{ Results } \\
\hline & KP97 & $x_{11}=1$ & Accepted \\
& AEA132 & $x_{12}=1$ & Accepted \\
& IST/NK & $x_{13}=0$ & No accepted \\
19h 20h & AF718/9 & $x_{14}=0$ & No accepted \\
& AF718/9 & $x_{15}=1$ & Accepted \\
\hline \multirow{4}{*}{ 20h 21h } & LH826 & $x_{21}=1$ & Accepted \\
& AF718/9 & $x_{22}=0$ & No accepted \\
& SN206 & $x_{23}=1$ & Accepted \\
& DN64 & $x_{24}=1$ & Accepted \\
& KP028 & $y_{21}=1$ & Accepted \\
& NST/NK & $y_{22}=1$ & Accepted \\
\hline \multirow{4}{*}{ 22h 23h } & DN64 & $x_{31}=1$ & Accepted \\
& QC376 & $x_{32}=1$ & Accepted \\
& KP97 & $y_{31}=1$ & Accepted \\
& AF718/9 & $y_{32}=1$ & Accepted \\
& AF718/9 & $y_{33}=0$ & No Accepted \\
& AF718/9 & $y_{34}=1$ & Accepted \\
& IB69 & $x_{33}=0$ & No accepted \\
\hline
\end{tabular}




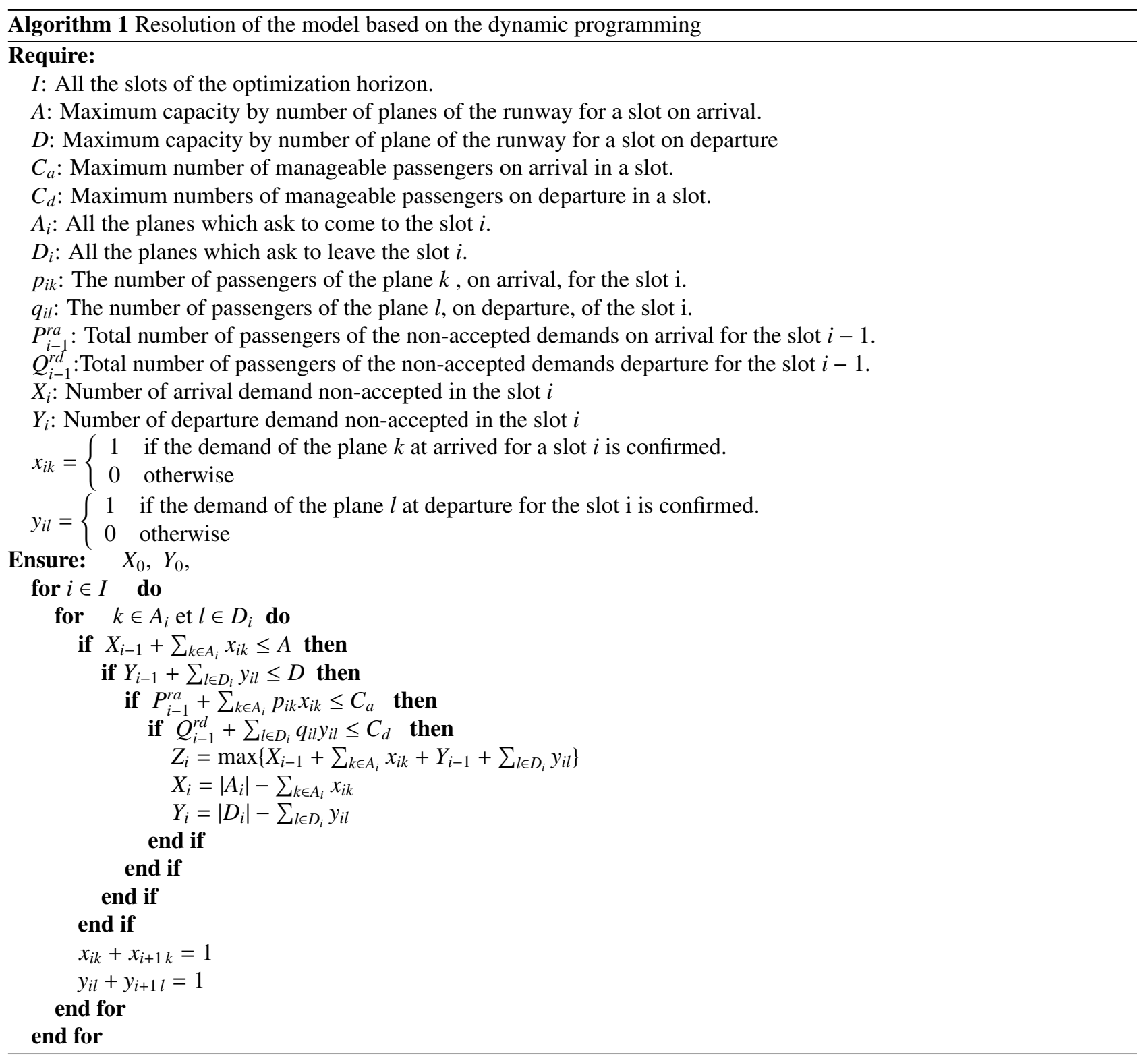

\section{Reference}

(1975). Capacité du système aéroportuaire - Manuel de capacité- pistes. Service Technique des Bases Aériennes: Juillet.

Alidou Sinare: Mthodologie d'valuation de la capacité de l'aire de mouvement et gestion automatique de l'aire de trafic. Application l'aroport de Dakar; Phd, (2007). EAMAC.

Gilbo, Eugene: Arrival/departure capacity trade off optimization: a case study at the st. louis lambert international airport (stl); (2003). Proc. of the Air Traffic Management R \& D Seminar.

Gilbo, Eugene P: Airport capacity: Representation, estimation, optimization; (1993). Control Systems Technology, IEEE Transactions, 1(3),144-154.

Idrissi, Abdellah, \& Li, Chu. Min: CSPAC: un modèle de résolution du problème d'allocation de capacités; MajecSTIC 2005: Manifestation des Jeunes Chercheurs francophones dans les domaines des STIC page 172-179, Université de picardie FRE. (2005).

Lenoir, Nathalie: Congestion et créneaux aéroportuaires; Colloque de la Commission pour l'Étude des Communautés Européennes; CEDECE. (2004).

Service technique de l'aviation civil: Capacits arogares; www.stac.aviation-civile.gouv.fr 
Isabelle: Comment quantifier et modliser le dimensionnement d'un composent d'un aroporté service technique de l'aviation civil. (2007). www.stac.aviation-cillvile.gouv.fr

Stoica, Dragos Constantin: Analyse, représentation et optimisation de la circulation des avions sur une plate-forme aéroportuaire; (2004). Institut National Polytechnique de Toulouse.

\section{Copyrights}

Copyright for this article is retained by the author(s), with first publication rights granted to the journal.

This is an open-access article distributed under the terms and conditions of the Creative Commons Attribution license (http://creativecommons.org/licenses/by/4.0/). 